\title{
Commentary
}

\section{Using IEC 61850 Protocol in Automation Systems of High Voltage}

\author{
Asghar Ehsani Fard ${ }^{1}$, Masoud Masomei ${ }^{2}$, Mehdi Hedayeti ${ }^{2}$, Hamid Chegini ${ }^{3}$ \\ ${ }^{1}$ Telecommunication of Non-profit Institution of Higher Education, ABA, Abyek, Qazvin, Iran \\ ${ }^{2}$ Non-profit Institution of Higher Education, ABA, Abyek, Qazvin, Iran \\ ${ }^{3}$ Research Assistant of Non-profit Institution of Higher Education, ABA, Abyek, Qazvin, Iran
}

\section{Email address:}

Ali.Ehsani_8520@gmail.com (A. E. Fard),massoud_masoumi@yahoo.com (M. Masomei), Mahdi-hedayati@yahoo.com (M. Hedayeti), Hamidchegini26@gmail.com (H. Chegini)

To cite this article:

Asghar Ehsani Fard, Masoud Masomei, Mehdi Hedayeti, Hamid Chegini. Using IEC 61850 Protocol in Automation Systems of High Voltage. Automation, Control and Intelligent Systems. Vol. 4, No. 2, 2016 pp. 28-34. doi: 10.11648/j.acis.20160402.14

Received: March 4, 2016; Accepted: March 16, 2016; Published: April 8, 2016

\begin{abstract}
Growing Development of new metric technology, communications on one side, and variety in IT and incremental information on the other side in designing and manufacturing automation station systems make the pioneers of this industry change the system with the aim of creating "a world, a technology, a standard" in this industry. This evolution led to create a new technique for designing automation systems which is called IEC 61850 standard. Considering the agreement on developing and using this standard, applying this in station automation systems was taken into account since 2005 . The new technology needs new interfaces to connect the high voltage switchgears such as modern current and voltage measurement transformers to station automation control systems. By introducing the new technology and using IEC 61850 protocols, designing automation systems with decentralized structure is possible and has a lot of advantages, however great care is needed in designing such system so that system reliability will not be impaired. In this thesis, for better understanding, we first explain briefly the old station structures and their transition and then the IEC 61850 standard structure will be investigated and this investigation includes the way of data transfer. Finally we refer to the effect of this standard on the structure of high voltage station automation system. In power distribution systems, IP and IEC-61850 are introduced as a security ring with more importance of communication, that in the meantime, IEC-61850 as the new international standard electrical station, is capable of providing the Integration, protection, control, measurement and monitoring functions within the stations with high-speed. So not only can prove the importance and the need to replace the IEC-61850 standard as the protocol with high speed data transfer capability ,but also we will also see reducing the time and power transmission losses in the automation network
\end{abstract}

Keywords: Station of Automation System, Protocol, IED, IEC 61850, Standard

\section{Introduction}

The new industries have complex activities and many functions inside themselves. They need a lot of control devices and intelligent electronic devices (IED) to have a safe activity. To operate automatically, these devices should work very harmoniously together. The IEC 61850 standard is a valid international one for building station communications and has created a new opportunity for evolution in system protection and control. This standard denotes a big step in making multi-objective intelligent electronic devices integrated based on development and implementation of advanced distributed protection and control procedures. To fulfill this evolution, it is necessary to know how to make use of it.

The advances in designing intelligent electronic devices and cooperative communication protocols like IEC61850 led to new generation of intelligent electronic devices. The new electronic intelligent devices, which are used to protect and control, can accept different voltage and current levels as their input and are capable of analyzing the data very quickly. The main advantage of using such devices which contain microprocessors is; 
i. Ease of connect to each other,

ii. Cost reduction of components,

iii. Maximization of system reliability

iiii. And capability of saving data extensively.

One effective method for using these intelligent electronic devices and reducing connections between them is using Goose messaging method in IEC61850 communication protocol which has a high speed and it is in a cooperative form. To understand the way of establishing connection between these intelligent electronic devices, automation design and station control, it is essential to be completely familiar with IEC 61850.

Communication protocol collection used in station level is one of important subjects in designing station automation system. This protocol needs to offer all required services which enable the optimal fulfillment of different station functions: The following are the most important necessities:

1- Appropriate definition of functional and applicable necessities,

2- Sufficient recognition of station communication protocols.

IEC61850 standard and its applicable programs are of important subjects in electricity due to being comprehensive and taking all control requirements and station protection into account.

\section{Theoretical Foundations of Research}

\subsection{The New Communicative Technologies}

A relatively new standard is IEC61850 in communicative networks of high voltage stations in which core element contains the following:

- A software module for every function in high voltage stations which is known as logical node and describes all available data.

- A definition of characteristics and way of communication between intelligent electronic devices in the station automation control system. Intelligent electronic devices refer to all devices that are available in the station computer network and exchange information with network which include numerical protection relays, BCU, station server computers and so on.

- A definition of programming language and configuration which is a tool for system engineering in automation stations and enables communication between tools and engineering software in systems. It can be used, for example, for system engineering in connection with dispatching center. It also facilitates definition of functions and special programs if necessary.

To draw the benefits of a standard, it is of high importance to satisfy present and future requirements and IEC61850 standard has this merit.

The main subject of this standard is designing a communicative system which provides interoperability of communication between functions in devices for different devices embedded in station produced by different manufacturers and meets all the needs well.

To achieve this, the functions are divided into sub functions which are known as logical nodes.

The logical nodes are 4 and core elements of information modules that should be exchanged between different devices in an automation system. In other words, an automation system includes exchange of logical nodes data with each other which is shown in figure 1.

As you see in Fig. 1, logical nodes including PDIS, TCTR, TVTR and XCBR are related to high voltage key.

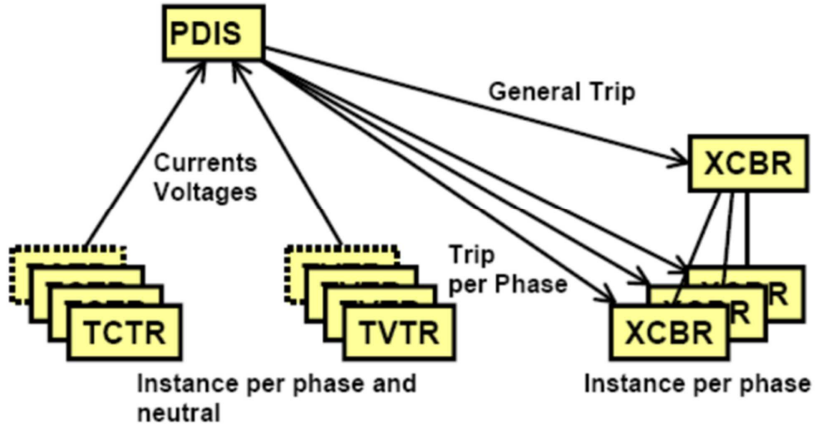

Fig. 1. Samples of logical needs exchange in a protection function.

As it is shown in Fig. 1, although different functions can be in separate physical devices depending on their respective manufacturer, all logical nodes can exchange information with each other through network if they are designed according to IEC61850 and so they can be produced by different manufacturers. This standard also defines logical and physical devices.

Logical devices are a collection of logical nodes that are collected in a joint group like protection group. Of course these logical devices are physically in a real device, e.g. protection relay and a protective relay has three logical devices, namely protection, control and measurement and the logical nodes, according to their functions, are in one of these logical devices. In figure 2, there are some examples of this model.

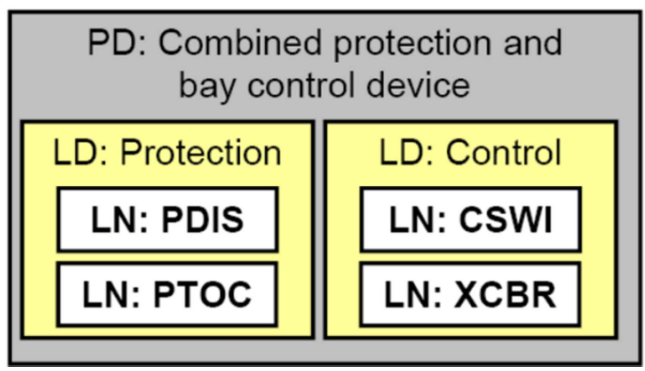

Fig. 2. An example of transformation between devices and logical nodes with physical devices.

The examined standard and structure of this and programming language are designed in such a way that they can support possible future functions. Also, it is necessary to separate the discussion of fast technology development from low speed of adding functions in station industry.

Every changes and optimization that occur in 
communications industry, including speed increasing that functions on protocol basis, will influence on 61850 IEC.

This separation of functions is done by an interface called ACSI2 that really maps the applied data by SCSM.3 The approach is shown by fig. 3 .

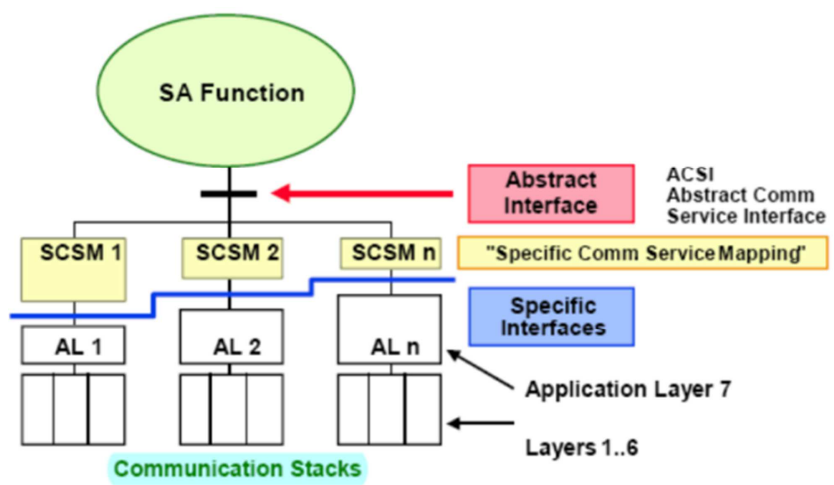

Fig. 3. A stack of information medium and the way of sending information to different parts of structure.

In fact the IEC 61850 carries out two separate mappings; one based on IEC 61850 for station passage (bus) that establishes a connection between Bay level intelligent electronic devices with station level or control room. This mapping is used for transforming sampled quantities which pass the seven-layers of ISO standard. And it does the second mapping that uses just two layers to increase speed.

This mapping is used to communicate between data layers and ISO standard physical layer in processing bus network which is between substation switchgear process level and bay level in modern automation systems.

\subsection{Automation Systems Levels with IEC 6180}

The modern automation designs of high voltage station are divided into three levels: processing level, bay level, and standard level. The station level communication generally refers to the control room devices and includes the network main servers (with bay level) and protection relays and bay controllers. The connection between them is done by station bus network in IEC-161850-8. In Figure 4, the three levels are shown.

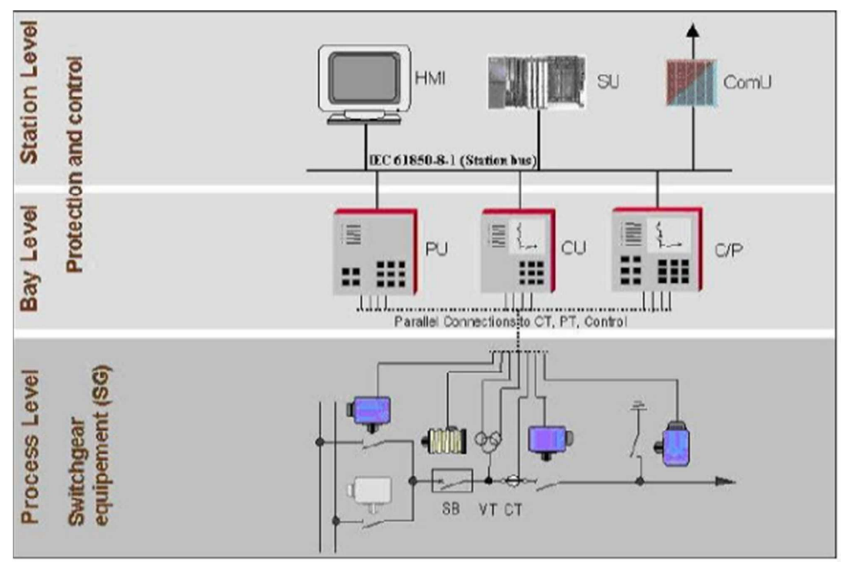

Fig. 4. The topology of station devices in three different levels.
The processing level devices generally include intelligent sensors and distant input outputs which has network and communication level called process bus network.

In other words, in stations which have modern automatic system with intelligent switchgear and have communication with the other devices, have two separated networks called station bus and process bus. As it is shown in Fig. 4, according to the other computer networks that function based on Ethernet protocols, the switchers of the two networks and information transform bus, have the role of Filter and responsibility of sending data to different parts of LAN network. If the station level node is likely to transform data with processing level, it will send its message through the network switches in bay level. Then the mentioned node will fulfill the message and send the result through the switch or connected unit, the responsibility of connected unit is mixing the information of 3-phase current and 3-phase voltage. This can also be the role of switch in bus network to transfer the information.

Many electronic devices that have different functions in the station are in the bay level and communicate with intelligent switchgear through the process bus network. In automation stations which are currently in our country, due to lack of modern switchgear the bay level communication is done by copper wires and cables.

Perhaps you consider that due to the presence of modern devices and their outcome, the communication network in processing level may decrease the quantity of consuming wiring and cables.

Basically, communication in processing bus network is similar to station bus communication, however there are two additional services for protecting bus network.

The first problem is quick and confident exchange of protection trip order between protection devices and switchgear, and the second one is quick transform of instance deals of electronic transformers for protection relays. These two services need quick execution on communication stacks and there should not be any delays.

So, the main selection is generally applying the quick networks in basic technology (processing bus) that must be executed in optical fiber communication.

\subsection{The New Technology in Primary Devices}

The new measurement transformers for current and intelligent voltage (optical current and voltage Trans) and also the electronic modern switchgears need new interfaces for automation systems. The new used technology in power key drivers, like new mechanism functions, which are controlled by serving motors, gives us this opportunity to have a direct communication with driver and breaker electronic mechanism.

The new serial interfaces with IEC61850 standard will provide communication. Using these keys with intelligent electronic abilities in switchgear with new interfaces will create a better situation for identifying diagnostic condition for observing breaker. This situation can be very useful for better exploitation and real time scheduling. 


\section{The Future Structure / Station Automation System}

The above mentioned technology, will direct the automation system toward decentralized structure and communication topology and this may lead to omission of cables and copper wires.

\subsection{Research Methodology}

Automation systems and numeric relays introduction with old switchgears, during the 15 years in our country, led us to a structure similar to figure 5 .

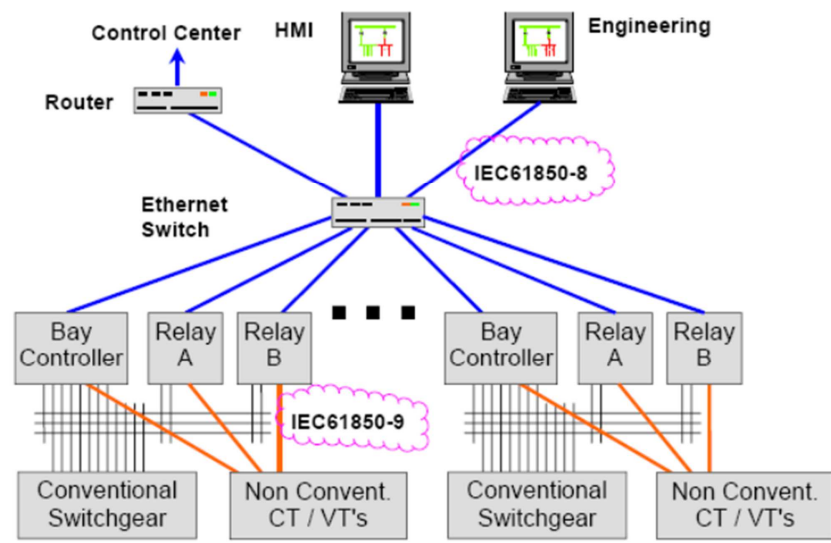

Fig. 5. Bus level and traditional wiring to controllers.

In the first step by entering the new transformers, the modern measurement of serial communication and point by point of these trances with relays will be possible.

Protection 61850-IEC and bay controllers will be created as shown in fig. 6 .

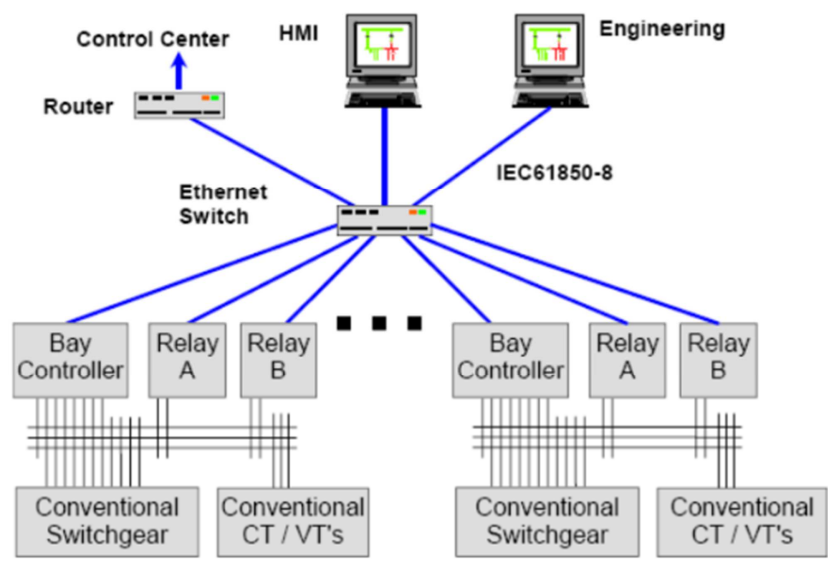

Fig. 6. Station bus and accurate transformers communication.

In the next step, the modern switchgears and keys with electronic drivers and mechanism, which have communication interfaces, will enter the market. They will have a structure as shown in fig. 7, the new bounty for automation systems which are called E C P or equipment close process. Processing to switchgears and high voltage devices are suitable for E C P.

E C P will create a communication network between switchgears and bay level and this will cause the omission of cables and copper wires and also the simplification of this part topology.

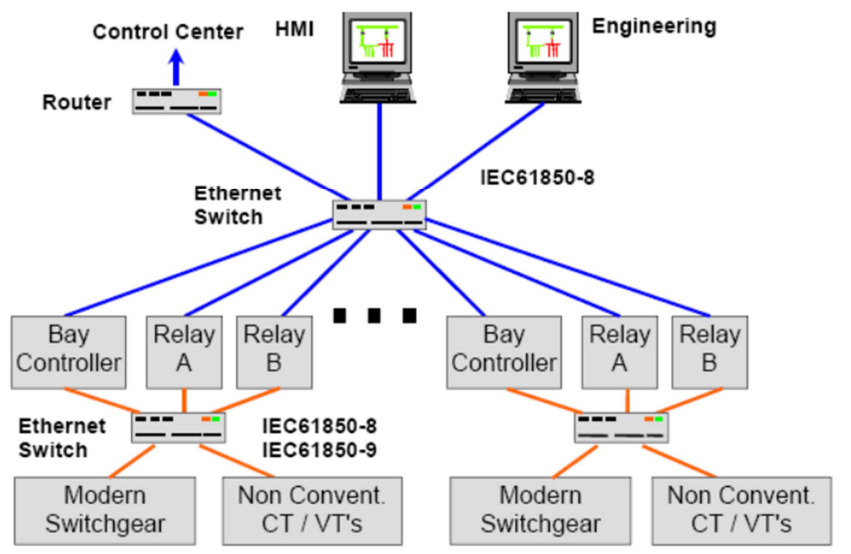

Fig. 7. The system structure by using the keys with mechanism and electronic drivers.

As 61850-8 IEC uses the equal communication topology for station and processing bus, the final structure will be as fig. 8 . The system will bring an akin structure for accessory to information.

In the system and also make it possible to use the structure and Ring Topology.

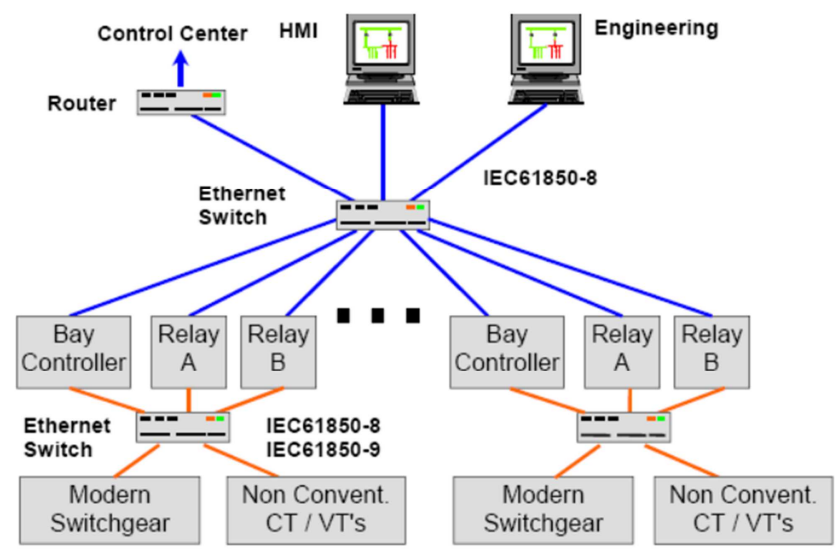

Fig. 8. The integrated communication network by using IEC 61850-9 and IEC 61850-8.

\subsection{The Details of the Close Processing Structure}

As discussed previously, there is a merging unit in the processing level that joins the current trances and voltage to the control and protection devices.

The output of this integration is according to 61850-9 IEC standard and current and voltage trances that are connected to the integration unit can be both conventional and traditional or the new intelligent one or a combination of the two kinds.

The output analog sample quantities of integration unit should be time sanction and have time tag.

This can be simultaneous with sampling which the total system dose for the total analog quantity or can have time tag for sampling itself. The processing structure of close device can be based on sending signals sent from switchgear and its 
integration method can be in the cable or copper wires or every modern system based on IEC 61850-9. For example, in Fig. 10, which is usual in our country, the integration of switchgear with bay level is in the form of copper wires. In addition to the presence of logical node connected to PDIS and PTOC, there is a controller device which includes logical nodes for controlling (CSWI, GCBR).

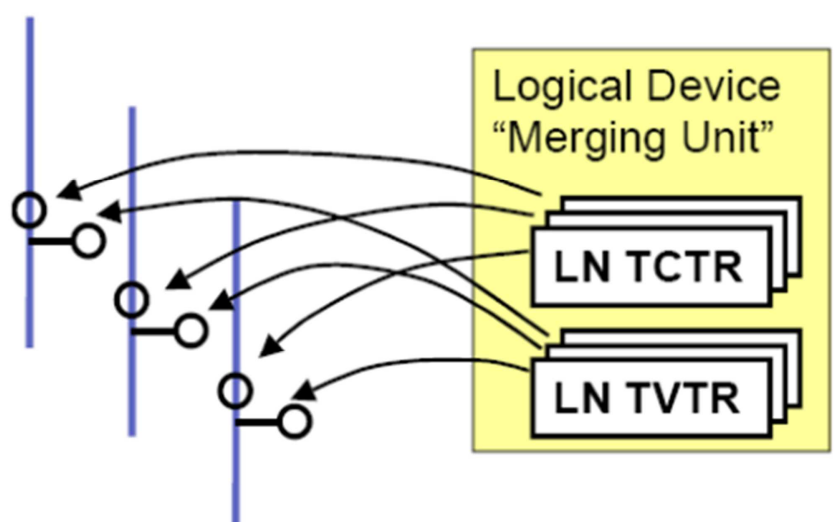

Fig. 9. The method of integration unit connected to line voltage and current trances.

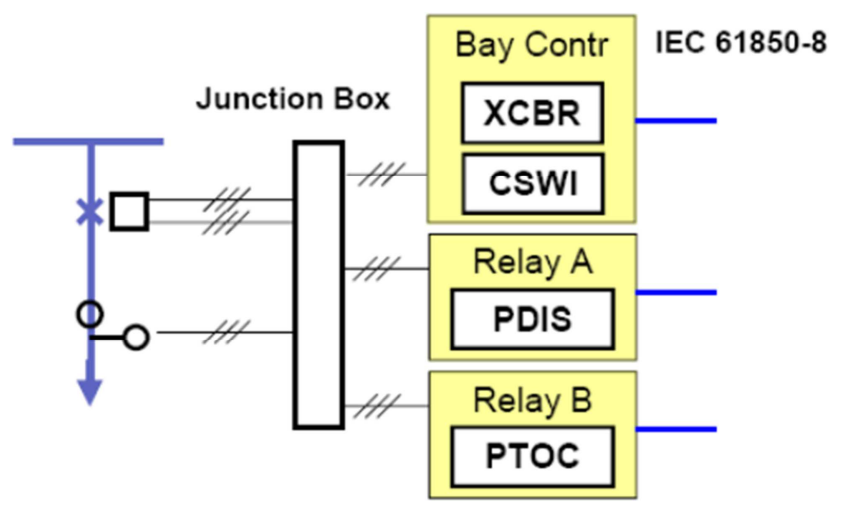

Fig. 10. The conventional connection of switchgears to lines.

In this structure there are a lot of cables and copper wires for each bay that need to be tested and wired. In the first step by entering the new modern measurement transformers which are connected to protection relays and buy controllers through combined units and connected to 61850-9 according to figure 11 .

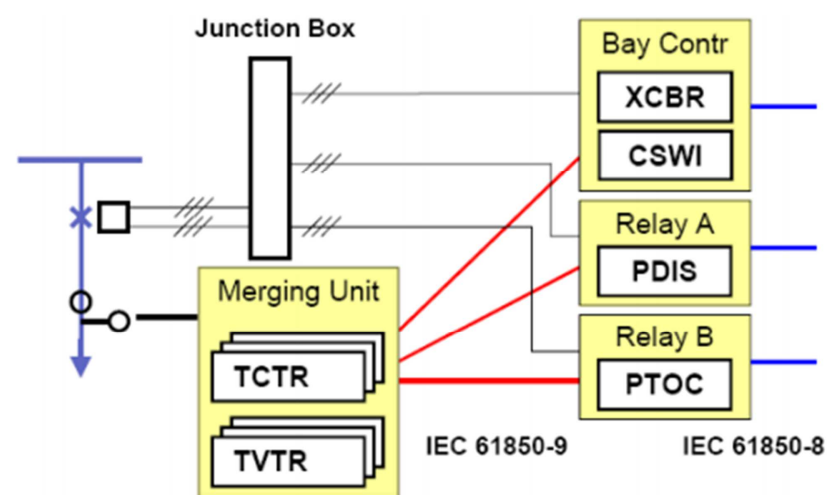

Fig. 11. The switcher connection (junction) to lines by using the modern tools transformers.
In the next step, addition to merging unit of modern sensors, there is a monitoring unit for electronic drivers of high voltage keys and the other devices, a switcher is added and made a new structure similar to fig. 12.

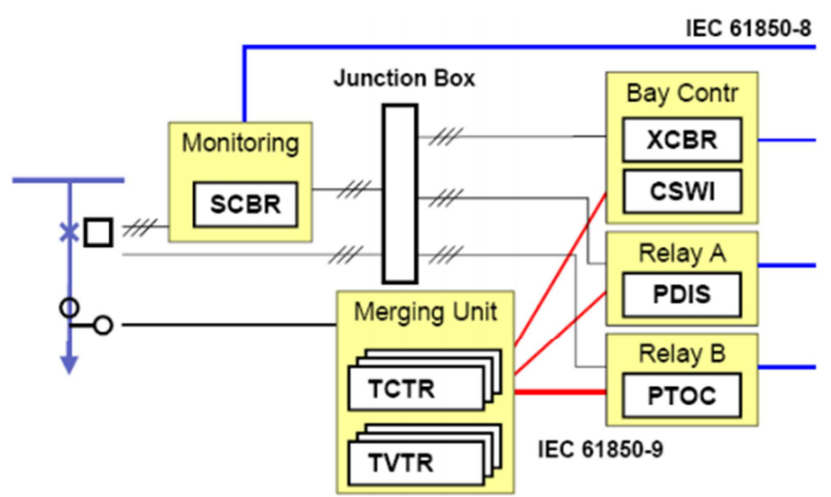

Fig. 12. Junction of monitoring unit for electronic control of high voltage keys.

The monitoring unit has a suitable communication with electronic sensor of breaker and also a wire and cable communication with control devices and protection relays that is used for sending information to station level according to $61850-8$ IEC. The commands of trip and alarm signals between breaker and protection relays are in copper cables and software form.

Finally by entering the fully intelligent switchgears, which is shown in fig. 13, monitoring and merging units are in network form and based on 61850-9 IEC and connected to bay devices, that is, by controllers and protection relays that are in copper cable and software form.

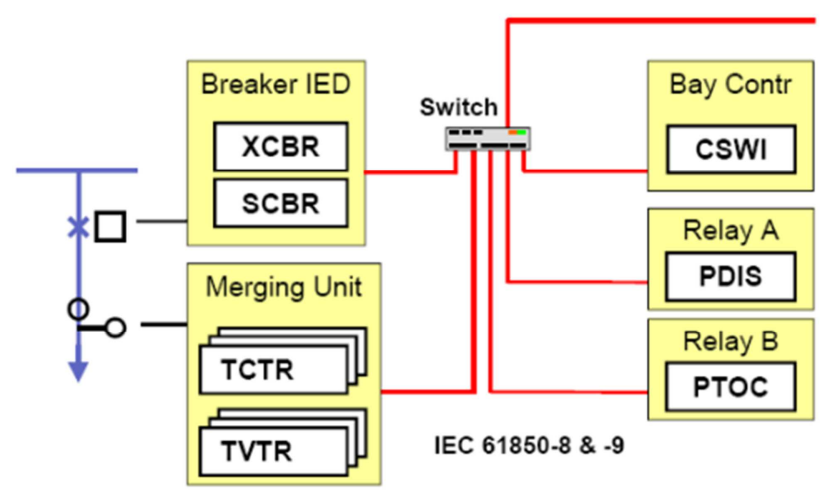

Fig. 13. Monitoring unit connection to merging unit in modern network.

In other words, processing bus network is used for junction between bay level and processing level and there is not any wire or cable junction between switchgear and protection relays and control devices.

\subsection{Function Integration}

Function integration in a set is an appropriate solution to decrease the number of devices and cost reduction. By decreasing the devices one can trust in system more. Today it is common to concentrate on all protection functions; control and monitoring in bay level and in separate devices in 
automation systems. By introducing intelligent switchgear systems and electronic sensors and merging units we can consider some integration. Look at fig. 14.

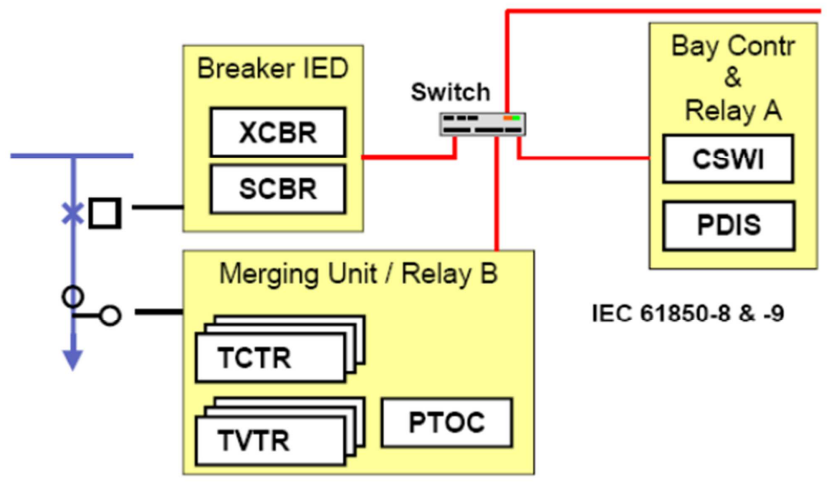

Fig. 14. Function integration merging at bay level and processing for entering.

Here by we should consider the following:

- Reliability of function ability of devices or system for fulfillment of functions of necessity times that directly refer to a parameter called MTBF.4 Considering the accessibility it the MTBF is larger and nearer to 100 percent it is a better one. In other words, when the distance is bigger, it means it is farther than problem.

Accessibility means getting access to the devices or system in the required time and it has opposite proportion to MTTR. That is, when the repair time is shorter, that means the situation is better.

The MTTR is dependent on power and diagnosis speed and knowledge at the problems. So the total equation and accessibility and reliability depend on identifying constant development of devices and self-examining of devices in the system and system itself. So, in modern systems, considering the numerical and intelligent devices even in processing level and omission of devices which are not visible, there is a better condition than in old systems.

In old systems there are not much support, additional devices like control devices, monitoring and protection of bases measurement. While in modern automation, there are many additional devices and hard ware for switchgear and other functions.

It seems that by introducing processing technology of modern systems which are close to switchgear level, there will be concern about reliability of function specially protection devices. This anxiety is at least limited to current systems and as mentioned above there are more function freedom. The freedom includes the following:

Design step, arrangement of place, control and protection function with self-examine ability, decreasing of devices.

So, there can be increasing of redundancy with respect to the importance at system.

In 115 key systems and similar systems we can connect the additional relays to current trances without the necessity of additional wiring. As the current transformers are connected to switchers and put their information in the network, several relays can use the information currently.

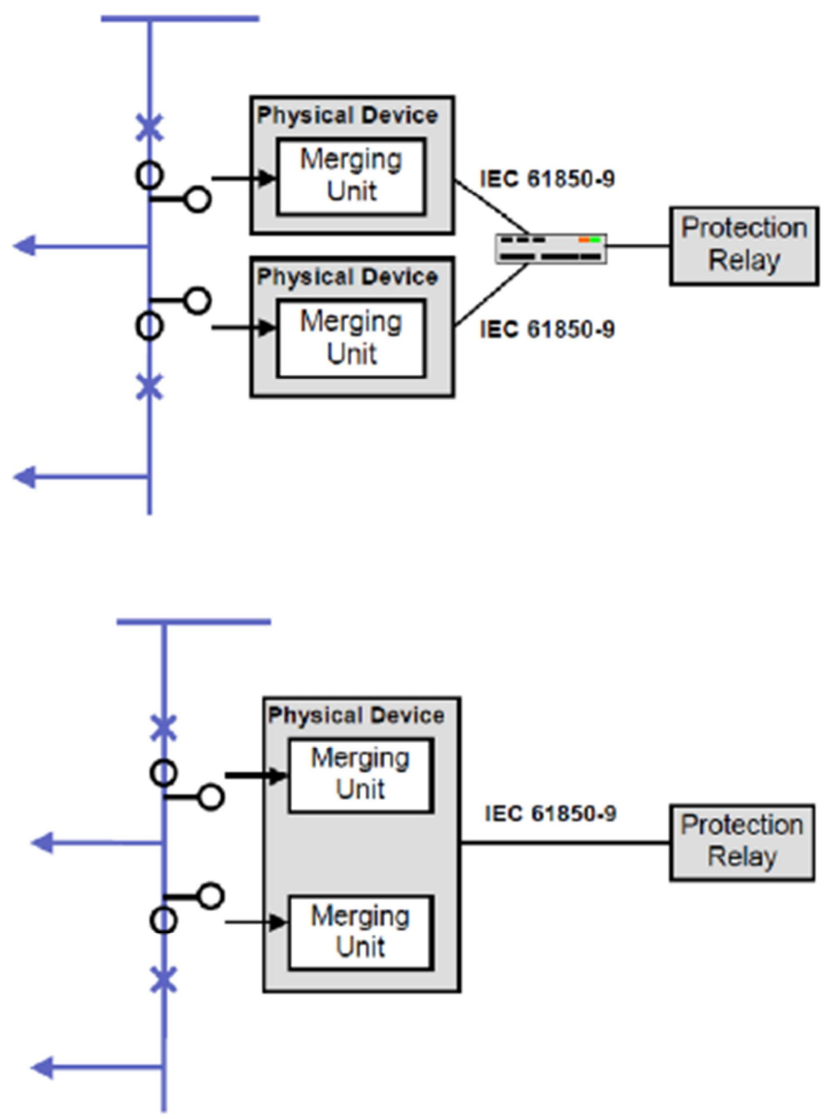

Fig. 15. Merging units junction in 1/5 key arrangement.

\section{Conclusion}

On the one hand, the results of this research reflect the replacement of the old generation with new posts that have many advantages. And the other is economically affordable. A typical life for a post, the payback time is only 10 years old. Thus, taking into account the complete life of post, alternative is economically quite affordable. The new processing technology of switchgear devices and 61850 IEC has a lot of advantages for high voltage station design. The advantages cause the omission of many copper wires and cables and consequently decreasing of work volume on wiring and testing process. The number of devices will decrease without self-examining ability and cause decreasing of system fouls and increasing of reliability.

Making support track at switchgear level for system and functions is becoming possible with acceptable cost and by integrating functions and number of devices, can lead to increase system reliability.

\section{References}

[1] Christoph Brunner, "How the IEC61850 support the migration phase" CIGRE 2002.

[2] Lars Andersson, "Discussion on cost differences between conventional and intelligent sensors as well as the different process connections for intelligent sensors", CIGRE 2002. 
[3] Ch. Brunner, G Schimmel, H. Schubert, "Standardisation of serial links replacing parallel wiring to transfer process data Approach, state and practical experience", CIGRE 2002 Paris.

[4] Lars Andersson, Klaus-Peter Brand, Wolfgang Wimmer, "The Impact of the coming Standard IEC61850 on the Life-cycle of Open Communication Systems in Substations", Distribution 2001, Transmission \& Distribution, Brisbane 2001.

[5] Lars Andersson, Klaus-Peter Brand, Wolfgang Wimmer. "The communication standard IEC61850 supports flexible and optimized substation automation architectures", 2nd International conference on Protection and Control in NEW DELHI 2001.

[6] C. Brunner, A. Ostermeier, "Serial Communication between Process And Bay Level - Standards And Practical Experience -", CIGRE 2000.

[7] M. Saitoh, T. Kimura, Y. Minami, N. Yamanka, S. Maruyama, T. Nakajima, M. Koskada, "Electronic Instrument Transformers for Integrated Substation Systems", IEEE/PES T\&D Conference and Exhibition 2002: Asia Pacific.

[8] Masayuki Kosakada, Hiroshi Watanabe, Tokuo Ito, Yoshito Sameda, Yuji Minami, Minoru Saito, Shiro Maruyama, "Integrated substation systems - harmonizing primary equipment with control and protection systems", IEEE/PES T\&D Conference and Exhibition 2002: Asia Pacific.
[9] IEC 61850 Communication Networks and systems in substations, IEC standard in ten main parts, first parts published in 2002.

[10] J. Rosenberg, H. Schulzrinne, G. Camarillo, A. Johnston, J. Peterson, R. Sparks, M. Handley, E. Schooler, "SIP: Session Initiation Protocol", RFC 3261, June 2002.

[11] J. Rosenberg, "A Framework for Conferencing with the Session Initiation Protocol (SIP)", RFC 4353, February 2006.

[12] R. Even, N. Ismail, "Conferencing Scenarios", RFC 4597, August 2006.

[13] http://www.iptel.org/sems.

[14] C. Partridge, "Isochronous Applications Do Not Require Jitter-Controlled Networks", RFC 1257, September 1991.

[15] H. Schulzrinne, S. Casner, R. Frederick, V. Jacobson, "RTP: A Transport Protocol for Real-Time Applications", RFC 3550, July 2003.

[16] A. H. Ashouri, F. Samsami, A. Akbari, "E-Learning Media Server Evaluation and its architecture modeling with signaling load tests," ICeLT, IUST, Tehran, Iran, Dec 2009.

[17] A. H. Ashouri. "Media Server Evaluation and Real-Time Tests" Iran University of Science and Technology, B. Sc Thesis, p46-61, Sep 2009. 